\title{
Geometrically smooth spline bases for data fitting and simulation
}

\author{
Ahmed Blidia $^{\mathrm{a}}$, Bernard Mourrain ${ }^{\mathrm{a}}$, Gang Xu ${ }^{\mathrm{b}}$ \\ ${ }^{a}$ Univ. Côte d'Azur, Inria, Aromath, Sophia Antipolis, France \\ ${ }^{b}$ School of Computer Science and Technology, Hangzhou Dianzi University, Hangzhou 310018, P.R China
}

\begin{abstract}
Given a topological complex $\mathcal{M}$ with glueing data along edges shared by adjacent faces, we study the associated space of geometrically smooth spline functions that satisfy differentiability properties across shared edges. We present new and efficient constructions of basis functions of the space of $G^{1}$-spline functions on quadrangular meshes, which are tensor product b-spline functions on each quadrangle and with b-spline transition maps across the shared edges. This new strategy for constructing basis functions is based on a local analysis of the edge functions, and does not depend on the global topology of $\mathcal{M}$. We show that the separability of the space of $G^{1}$ splines across an edge allows to determine the dimension and a basis of the space of $G^{1}$ splines on $\mathcal{M}$. This leads to explicit and effective constructions of basis functions attached to the vertices, edges and faces of $\mathcal{M}$.

This basis construction has important applications in geometric modeling and simulation. We illustrate it by the fitting of point clouds by $G^{1}$ splines on quadrangular meshes of complex topology and in Isogeometric Analysis methods for the solution of diffusion equations. The ingredients are detailed and experimentation results showing the behavior of the method are presented.
\end{abstract}

\section{Introduction}

To describe and analyze shapes with complex topologies, one often starts with a coarse representation $\mathcal{M}$ that captures the topology and the principal geometric features of the shape. This representation can then be refined and tuned to describe more accurately the actual shape. If the coarse model is a mesh, a classical strategy to obtain a better approximation of the shape is to refine the mesh, by splitting some of its faces. This approach yields piecewise linear representations of the shape, which may require several levels of subdivisions in regions with high curvatures, in order to obtain a good approximation of the shape.

In this paper, we investigate a different strategy to compute accurate shape representations. Instead of splitting the coarse piecewise linear model, we increase the degree of the representation on each face of $\mathcal{M}$ with the aim to obtain better approximation performances with higher order of convergence. More precisely, we investigate the problem of constructing efficiently families of regular functions which are piecewise polynomial on each face of the coarse mesh $\mathcal{M}$ and with regularity properties across the edges shared by two faces. We will assume that all faces of $\mathcal{M}$ are quadrangular faces and that tensor product b-spline functions of the same degree and the same knot vector are used on each face of $\mathcal{M}$. The regularity that we impose across the edges shared by two faces is the continuity of the tangent planes of the parameterizations. This corresponds to geometrically smooth spline functions (as opposed to parametrically smooth spline functions), also called $G^{1}$ spline functions. Our aim is to analyse in details the space of $G^{1}$ splines on an arbitrary quad mesh and to compute efficiently bases which are suitable for fitting and numerical simulation problems.

Many works over the last decades have been investigating the problem of constructing $G^{1}$ surfaces from (quad) meshes. This includes subdivision surface constructions Catmull-Clark (1978), macro patch

Email addresses: ahmed.blidia@inria.fr (Ahmed Blidia), bernard.mourrain@inria.fr (Bernard Mourrain), gxu@hdu.edu.cn (Gang Xu) 
constructions in low degree Loop (1994b), Peters (1995), Prautzsch (1997), Reif (1995), Peters (2002), Ying et al. (2004), Fan et al. (2008), Hahmann et al. (2008), Bonneau et al. (2014), manifold based constructions Gu et al. (2006), He et al. (2006), Tosun et al. (2011), Wang et al. (2016), constructions using transition maps defined from mesh embeddings Beccari et al. (2014), or constructions using guided surfaces Karčiauskas et al. (2016), Karčiauskas et al. (2017a), Karčiauskas et al. (2018). Some of these works focus on the construction of $\mathrm{G}^{1}$ spline surfaces that interpolate a network of curves Sarraga (1987), Sarraga (1989), Peters (1991), Loop (1994a), Tong et al. (2009), Cho et al. (2006), Bonneau et al. (2014), Karčiauskas et al. (2017b), Karčiauskas et al. (2018). To solve this so-called transfinite interpolation problem, vertex enclosure constraints have to be satisfied by the curves at a vertex of even valency. Explicit rational transition maps of degree 1 or 2 have been used in some of these constructions Hahmann et al. (2008), Peters et al. (2010), Beccari et al. (2014), Bonneau et al. (2014), Karčiauskas et al. (2016), Karčiauskas et al. (2017b), Karčiauskas et al. (2018). They depend on the valence of the end points of an edge and on its proximity to an extraordinary vertex. In Peters et al. (2010), it is shown that rational transition maps of degree 1 should not be used if extraordinary vertices are separated by ordinary vertices.

The analysis of spaces of $\mathrm{G}^{1}$ spline functions on general meshes is a problem, which has been much less investigated. Several approaches have been considered to construct these spaces. One of them consists in using a parametrization of a given domain to deduce transition maps that define the regularity of the spline functions across shared edges. More precisely, the $\mathrm{G}^{1}$ regularity condition is the continuity of the tangent planes of the graph surface associated to the function and the domain parametrization. It is assumed that the domain is planar. The resulting transition maps across shared edges involve two-by-two determinants of the gradients of the parametrizations coordinates along the edge. This approach is investigated for instance in Kapl et al. (2015), Kapl et al. (2017a), Bercovier et al. (2017), Kapl et al. (2017b) for piecewise bilinear parametrizations of planar quad meshes and for parametrizations of higher degree in Collin et al. (2016), Kapl et al. (2019). In Chan et al. (2018), this approach is extended to non-planar parametrizations, involving the first fundamental form associated to the parameterization and thus increasing the degree of the transition maps. A locking phenomenon on the isogeometric solutions of elliptic problems is observed in numerical experimentations, with transitions maps of high degree compared to the degree of freedom of the spline spaces.

The construction of bases for the corresponding space of $\mathrm{G}^{1}$ functions are either obtained by solving the linear system deduced from the sampled $\mathrm{G}^{1}$ constraints or in Bercovier et al. (2017), Kapl et al. (2017b), by explicitly computing Minimal Determining Sets (MDS) of coefficients in the case of bi-degree 3 and 4 polynomial splines on planar quadrangular meshes with bilinear parametrization.

A second approach to analyse $G^{1}$ spline spaces is to start from transition maps and to use the $G^{1}$ functions associated to these transition maps, for the domain parametrization and the numerical simulation. This is the approach followed in Mourrain et al. (2016), or Blidia et al. (2017) and that we will also adopt in this paper. To obtain $\mathrm{G}^{1}$ functions which are not degenerate, the transition maps should satisfy compatibility conditions around vertices. Spaces of degenerate $\mathrm{G}^{1}$ spline functions where these compatibility conditions are not satisfied, have also been exploited in IsoGeometric Analysis for instance in Wu et al. (2017), Toshniwal et al. (2017).

Dimension formulae for spaces of $\mathrm{G}^{1}$-splines on meshes are provided, for instance, in Bercovier et al. (2017), Kapl et al. (2017a), for planar quadrangular meshes under some non-degeneracy restrictions, and for general quadrangular meshes in Mourrain et al. (2016), Blidia et al. (2017).

The fact that geometrically continuous constructions yield parametrically continuous isogeometric functions Groisser et al. (2015) has been exploited in isogeometric methods that compute a full basis of the $\mathrm{G}^{1}$ spline spaces. Functions associated to the vertices of the coarse mesh in geometrically smooth surface constructions Karčiauskas et al. (2016), Majeed et al. (2017) have also been used in isogeometric approaches, but with non-optimal convergence rates.

In this paper, we present new constructions of $\mathrm{G}^{1}$-spline bases on quadrangular meshes $\mathcal{M}$ of arbitrary topology with b-spline representations of given bi-degree and knots subdivisions on each face. We assume that the transition maps across shared edges are given by rational b-spline functions and satisfy compatibility conditions around a vertex. We describe and use such transition maps of low degree, which depend only on the valence of the end points of the edge in the quad mesh $\mathcal{M}$. 
The new strategy that we propose to analyse of the complete space of $\mathrm{G}^{1}$-spline functions on $\mathcal{M}$ is based on a local analysis of the $\mathrm{G}^{1}$ edge functions and does not rely on the global topology of $\mathcal{M}$. We show that if a separability condition is satisfied for each edge, the dimension and a basis of the whole space of $\mathrm{G}^{1}$-splines can be deduced from this analysis of $\mathrm{G}^{1}$-splines along the edges.

We adapt the construction of Minimal Determining Sets (see e.g. Lai et al. (2007), Alfeld (2000)) of coefficients to the $\mathrm{G}^{1}$ edge functions and show how to deduce efficiently basis functions for the corresponding $\mathrm{G}^{1}$ space, based on local linear operations associated to the edges of the mesh. In this approach, the vertex enclosure constraints at vertices of even valence are implicitly taken into account.

This new construction yields basis functions attached respectively to vertices, edges and faces. We also obtain a dimension formula for the space of $\mathrm{G}^{1}$-splines, depending on the number of vertices, edges and faces, and the properties of the glueing data at the end points of the shared edges.

This basis construction is exploited in two types of applications. The first problem is the fitting of point clouds by $\mathrm{G}^{1}$-splines on quadrangular meshes. The second one is the solution of diffusion equations, following an Isogeometric Analysis approach, based on the $\mathrm{G}^{1}$-spline basis functions.

The remainder of the paper is structured as follows. The definition of $\mathrm{G}^{1}$-Spline spaces and the properties of transition maps are presented in Section 2. The construction of $\mathrm{G}^{1}$-Spline basis is described in Section 3. Section 4 presents the application to point clouds fitting by $\mathrm{G}^{1}$-splines on quadrangular meshes. Section 5 presents the application of $\mathrm{G}^{1}$-splines in isogeometric analysis. Finally, this paper is concluded and future work is outlined in Section 6.

\section{Space of $\mathrm{G}^{1}-\mathrm{Splines}$}

In this section, we present the general notions we use to define $\mathrm{G}^{1}$ spline spaces and the properties of the transition maps that we require. To define $G^{1}$-splines, we first need a topological surface $\mathcal{M}$ given by

- a collection $\mathcal{M}_{2}$ of (planar) polygons, called faces of $\mathcal{M}$,

- a collection of homeomorphisms $\phi_{\sigma_{i}, \sigma_{j}}: \tau_{i} \mapsto \tau_{j}$ between polygonal edges from different polygons $\sigma_{i}$ and $\sigma_{j}$ of $\mathcal{M}_{2}$

where a polygonal edge can be glued with at most one other polygonal edge, and it cannot be glued with itself. The shared edges (resp. the points of the shared edges) are identified with their image by the corresponding homeomorphism. The collection of edges (resp. vertices) is denoted $\mathcal{M}_{1}$ (resp. $\mathcal{M}_{0}$ ).

Hereafter, we will consider only quadrangular faces with 4 edges, which we identify with the unit square $[0,1]^{2} \subset \mathbb{R}^{2}$.

Then, we need glueing data on the topological surface $\mathcal{M}$, which consists of the following:

- for each edge $\tau \in \mathcal{M}_{1}$ of a cell $\sigma$, an open set $U_{\tau, \sigma}$ of $\mathbb{R}^{2}$ containing $\tau$;

- for each edge $\tau \in \mathcal{M}_{1}$ shared by two polygons $\sigma_{i}, \sigma_{j} \in \mathcal{M}_{2}$, a $\mathrm{C}^{1}$-diffeomorphism called the transition map $\phi_{\sigma_{j}, \sigma_{i}}: U_{\tau, \sigma_{i}} \rightarrow U_{\tau, \sigma_{j}}$ between the open sets $U_{\tau, \sigma_{i}}$ and $U_{\tau, \sigma_{j}}$, and its corresponding inverse map $\phi_{\sigma_{i}, \sigma_{j}}$;

This notion of glueing data is the same as the one used for instance in Mourrain et al. (2016), Blidia et al. (2017), Kapl et al. (2019). The idea of using glueing data is coming from the theory of manifolds and the construction of abstract varieties (c.f. e.g. the work of Weil (1946)).

For an edge $\tau$ shared by two polygons $\sigma_{0}, \sigma_{1} \in \mathcal{M}_{2}, \tau=\tau_{0}$ in $\sigma_{0}, \tau=\tau_{1}$ in $\sigma_{1}$ respectively, the transition map $\phi_{\sigma_{0}, \sigma_{1}}$ between the two cells is, in suitable frames, of the form:

$$
\phi_{\sigma_{0}, \sigma_{1}}:\left(u_{1}, v_{1}\right) \longrightarrow\left(u_{0}, v_{0}\right)=\left(\begin{array}{c}
v_{1} \mathfrak{b}_{\tau}\left(u_{1}\right)+v_{1}^{2} \rho_{1}\left(u_{1}, v_{1}\right) \\
u_{1}+v_{1} \mathfrak{a}_{\tau}\left(u_{1}\right)+v_{1}^{2} \rho_{2}\left(u_{1}, v_{1}\right)
\end{array}\right)
$$

where $\mathfrak{a}_{\tau}\left(u_{1}\right), \mathfrak{b}_{\tau}\left(u_{1}\right), \rho_{1}\left(u_{1}, v_{1}\right), \rho_{2}\left(u_{1}, v_{1}\right)$ are $C^{1}$ functions. The shared edge is defined by $v_{1}=0$ on $\sigma_{1}$ and by $u_{0}=0$ on $\sigma_{0}$. The functions $\left[\mathfrak{a}_{\tau}, \mathfrak{b}_{\tau}\right]$ are called the glueing data along $\tau$ on $\sigma_{1}$. We assume we are given 
this set $\mathfrak{g}$ of glueing data $\left[\mathfrak{a}_{\tau}, \mathfrak{b}_{\tau}\right]$ for all shared edges of $\mathcal{M}$. As we will see, it completely specifies the set of $\mathrm{G}^{1}$-splines $\mathcal{M}$.

The differentiable functions on $\mathcal{M}$ are collections $f=\left(f_{\sigma}\right)_{\sigma \in \mathcal{M}_{2}}$ of differentiable functions on each face $\sigma \in \mathcal{M}_{2}$ such that for any two faces $\sigma_{0}$ and $\sigma_{1}$ sharing an edge $\tau$ with $\phi_{0,1}$ as transition map, the two functions $f_{\sigma_{1}}$ and $f_{\sigma_{0}} \circ \phi_{0,1}$ have the same Taylor expansion of order 1 .

This leads to the following two relations for each $u_{1} \in[0,1]$ :

$$
\begin{aligned}
f_{1}\left(u_{1}, 0\right) & =f_{0}\left(0, u_{1}\right) \\
\frac{\partial f_{1}}{\partial v_{1}}\left(u_{1}, 0\right) & =\mathfrak{b}_{\tau}\left(u_{1}\right) \frac{\partial f_{0}}{\partial u_{0}}\left(0, u_{1}\right)+\mathfrak{a}_{\tau}\left(u_{1}\right) \frac{\partial f_{0}}{\partial v_{0}}\left(0, u_{1}\right)
\end{aligned}
$$

where $f_{1}=f_{\sigma_{1}}, f_{0}=f_{\sigma_{0}}$ are the restrictions of $f$ on the faces $\sigma_{0}, \sigma_{1}$.

To define the space of $\mathrm{G}^{1}$-splines on $\mathcal{M}$, we will choose each face restriction $f_{\sigma}$ to be an element of the space $\mathcal{R}_{d, \mathbf{t}}$ a tensor product b-spline with open knot vector $\mathbf{t}=\left[t_{1}, \ldots, t_{s}\right] \subset \mathbb{R}$ of degree $d$ in each of the variables $u$ and $v$ (the first and last knots are repeated $d+1$ times). An element $f_{\sigma} \in \mathcal{R}_{d, \mathbf{t}}$ is of the form

$$
f_{\sigma}:=\sum_{1 \leqslant i, j \leqslant m} c_{i, j}^{\sigma}\left(f_{\sigma}\right) b_{i}\left(u_{\sigma}\right) b_{j}\left(v_{\sigma}\right)
$$

where $c_{i, j}^{\sigma}\left(f_{\sigma}\right) \in \mathbb{R}$ and $b_{1}, \ldots, b_{m}$ are the b-spline basis functions of the space $\mathcal{U}_{d, \mathbf{t}}$ of splines of degree $d$ and knots $\mathbf{t}$ in one variable $u$. We denote by $\left(b_{i, j}^{\sigma}\right)_{0 \leqslant i, j \leqslant m-1}$ the b-spline basis functions on the face $\sigma$. With the previous notation, $b_{i, j}^{\sigma}=b_{i}\left(u_{\sigma}\right) b_{j}\left(v_{\sigma}\right)$. The functions are represented by a vector $\mathbb{R}^{m^{2} \times\left|\mathcal{M}_{2}\right|}$ with 1 at the position corresponding to the coefficient $c_{i, j}^{\sigma}$ and 0 elsewhere.

We will consider hereafter glueing data $\left[\mathfrak{a}_{\tau}, \mathfrak{b}_{\tau}\right]$, which are spline functions $\in \mathcal{U}_{d^{\prime}, \mathbf{t}^{\prime}}$ of degree $d^{\prime}$ and knots $\mathbf{t}^{\prime}=\left[t_{1}^{\prime}, \ldots, t_{s^{\prime}}^{\prime}\right] \subset \mathbb{R}$, such that $t_{1}^{\prime}=\cdots=t_{d^{\prime}}^{\prime}$ and $t_{s^{\prime}-d^{\prime}}^{\prime}=\cdots=t_{s^{\prime}}^{\prime}$.

Definition 2.1. We denote by $\mathcal{S}_{d, \mathbf{t}}(\mathcal{M}, \mathfrak{g})$ the vector space of differentiable functions on $\mathcal{M}$ for the glueing data $\mathfrak{g}$, with face restrictions $f_{\sigma}$ in $\mathcal{R}_{d, \mathbf{t}}$.

An element in $\mathcal{S}_{d, \mathbf{t}}(\mathcal{M}, \mathfrak{g})$ is in the space $\mathcal{R}_{d, \mathbf{t}}(\mathcal{M})$ of b-spline functions on each face. It will be represented by its b-spline coefficients on each face, that is, by a vector in $\mathbb{R}^{m^{2} \times\left|\mathcal{M}_{2}\right|}$.

For two vectors $f, f^{\prime} \in \mathcal{R}_{d, \mathbf{t}}(\mathcal{M}) \equiv \mathbb{R}^{m^{2} \times\left|\mathcal{M}_{2}\right|}$, we denote by $\left\langle f, f^{\prime}\right\rangle$ the usual scalar product of their b-spline coefficients.

For a vertex $\gamma$ of a face $\sigma$, we denote by $T_{\gamma}^{\sigma}$ the map $T_{\gamma}^{\sigma}: \mathcal{S}_{d, \mathbf{t}}(\mathcal{M}, \mathfrak{g}) \rightarrow \mathbb{R}^{4}$ that associates to each differentiable function $f \in \mathcal{S}_{d, \mathbf{t}}(\mathcal{M}, \mathfrak{g})$ the following vector:

$$
T_{\gamma}^{\sigma}(f)=\left[c_{0,0}^{\sigma}(f), c_{1,0}^{\sigma}(f), c_{0,1}^{\sigma}(f), c_{1,1}^{\sigma}(f)\right]
$$

where $c_{0,0}, c_{1,0}, c_{0,1}, c_{1,1}$ are the corner b-spline coefficients of $f \in \mathcal{R}_{d, \mathbf{t}}$ corresponding to $\gamma$. We call these coefficients, the (first) Taylor coefficients of $f$ around $\gamma$. For $\gamma \in \mathcal{M}_{0}$ an end point of an edge $\tau$ shared by the faces $\sigma_{0}, \sigma_{1}$, let

$$
T_{\gamma}^{\tau}:\left(f_{0}, f_{1}\right) \mapsto T_{\gamma}^{\sigma_{0}}\left(f_{0}\right) \oplus T_{\gamma}^{\sigma_{1}}\left(f_{1}\right) .
$$

A desired property for the space of $\mathrm{G}^{1}$-splines is the possibility to arbitrarily fix the Taylor coefficients at a vertex on a face. This means that at each vertex, we should be able to fix the values, derivatives and cross derivatives and construct a $\mathrm{G}^{1}$-spline function that interpolates these values and derivatives. This leads to the following definition:

Definition 2.2. The space $\mathcal{S}(\mathcal{M}, \mathfrak{g})$ of $G^{1}$-spline space is ample if for every vertex $\gamma \in \mathcal{M}_{0}$ and every face $\sigma \in \mathcal{M}_{2}$ adjacent to $\gamma$, the map $T_{\gamma}^{\sigma}$ is surjective.

To get an ample space of $\mathrm{G}^{1}$-splines on $\mathcal{S}_{\gamma}$, we assume that the glueing data satisfy the following condition: 
Condition 2.3 (cocycle condition). For each interior vertex $\gamma$ of valence $v$ on the faces $\sigma_{1}, \ldots, \sigma_{v}$, such that $\sigma_{i}$ and $\sigma_{i+1}$ share the edge $\tau_{i}$ for $i \in 1 \ldots v-1$ and $\sigma_{v}, \sigma_{1}$ share the edge $\tau_{v}$.

$$
\prod_{i=1}^{v}\left(\begin{array}{cc}
0 & 1 \\
\mathfrak{b}_{\tau_{i}}(0) & \mathfrak{a}_{\tau_{i}}(0)
\end{array}\right)=\left(\begin{array}{ll}
1 & 0 \\
0 & 1
\end{array}\right) .
$$

where $\left(\mathfrak{a}_{\tau_{i}}, \mathfrak{b}_{\tau_{i}}\right)$ for $i \in 1 \ldots v$ are the glueing data used for the junction along the edge $\tau_{i}$.

Additionally if $v=4$ and $\mathfrak{a}_{\tau_{i}}(0)=0$, the glueing data $\left(\mathfrak{a}_{\tau_{i}}, \mathfrak{b}_{\tau_{i}}\right), i=1 \ldots 4$ at the vertex $\gamma$, called a crossing vertex, must also satisfy

$$
\begin{aligned}
& \mathfrak{a}_{\tau_{1}}^{\prime}(0)+\frac{\mathfrak{b}_{\tau_{4}}^{\prime}(0)}{\mathfrak{b}_{\tau_{4}}(0)}=-\mathfrak{b}_{\tau_{1}}(0)\left(\mathfrak{a}_{\tau_{3}}^{\prime}(0)+\frac{\mathfrak{b}_{\tau_{2}}^{\prime}(0)}{\mathfrak{b}_{\tau_{2}}(0)}\right) \\
& \mathfrak{a}_{\tau_{2}}^{\prime}(0)+\frac{\mathfrak{b}_{\tau_{1}}^{\prime}(0)}{\mathfrak{b}_{\tau_{1}}(0)}=-\mathfrak{b}_{\tau_{2}}(0)\left(\mathfrak{a}_{\tau_{4}}^{\prime}(0)+\frac{\mathfrak{b}_{\tau_{3}}^{\prime}(0)}{\mathfrak{b}_{\tau_{3}}(0)}\right)
\end{aligned}
$$

(see e.g. Peters et al. (2010), Mourrain et al. (2016), Blidia et al. (2017)).

\section{Construction of $\mathrm{G}^{1}$-spline basis}

\subsection{G-splines along an edge}

We consider first a topological mesh $\mathcal{M}_{\tau}$ with two faces $\sigma_{0}, \sigma_{1}$ sharing an edge $\tau$, with the glueing data $\mathfrak{g}_{\tau}=(\mathfrak{a}, \mathfrak{b})$.

The $\mathrm{G}^{1}$-spline functions of $\mathcal{S}_{d, \mathbf{t}}\left(\mathcal{M}_{\tau}, \mathfrak{g}_{\tau}\right)$ are the pairs $f=\left(f_{0}, f_{1}\right)$ of b-spline functions $f_{0}, f_{1} \in \mathcal{R}_{d, \mathbf{t}}$, which satisfies the relations (2) and (3). If $\tau$ is defined by $v_{1}=0$ on $\sigma_{1}$ and $u_{0}=0$ on $\sigma_{0}$, these relations involve only the b-spline coefficients $c_{i, j}^{\sigma_{1}}(f), c_{j, i}^{\sigma_{0}}(f)$ for $0 \leqslant i \leqslant m-1$ and $0 \leqslant j \leqslant 1$. The other coefficients can be chosen arbitrarily. Let us denote by $\mathcal{S}_{\tau}$ the space of b-spline functions $\left(f_{0}, f_{1}\right)$ in $\mathcal{S}_{d, \mathbf{t}}\left(\mathcal{M}_{\tau}, \mathfrak{g}_{\tau}\right)$ with all these other coefficients equal to 0 . The elements of $\mathcal{S}_{\tau}$ are the $\mathrm{G}^{1}$-spline functions supported along the edge $\tau$.

Let $\gamma, \gamma^{\prime} \in \mathcal{M}_{0}$ be the end vertices of $\tau$. We denote $\mathcal{E}_{\tau}=\mathcal{S}_{\tau} \cap \operatorname{ker} T_{\gamma}^{\tau} \cap \operatorname{ker} T_{\gamma^{\prime}}^{\tau}$ (see definition (4)). It is the vector space of $\mathrm{G}^{1}$-spline in $\mathcal{S}_{\tau}$ supported along $\tau$, with zero b-spline coefficients at $\gamma$ and $\gamma^{\prime}$.

Let $\mathcal{E}_{\tau}^{\perp}$ be the b-splines in $\mathcal{R}_{d, \mathbf{t}}\left(\mathcal{M}_{\tau}\right)$ which are orthogonal to all the elements in $\mathcal{E}_{\tau}$ for the scalar product on the b-spline coefficients. We denote $\mathcal{S}_{\gamma, \tau}=\mathcal{S}_{\tau} \cap \mathcal{E}_{\tau}^{\perp} \cap \operatorname{ker} T_{\gamma^{\prime}}^{\tau}$ and similarly $\mathcal{S}_{\gamma^{\prime}, \tau}=\mathcal{S}_{\tau} \cap \mathcal{E}_{\tau}^{\perp} \cap \operatorname{ker} T_{\gamma}^{\tau}$. By construction, we have

$$
\mathcal{S}_{\tau} \supset \mathcal{S}_{\gamma, \tau} \oplus \mathcal{E}_{\tau} \oplus \mathcal{S}_{\gamma^{\prime}, \tau} .
$$

Definition 3.1. The space $\mathcal{S}_{\tau}$ is separable if $\mathcal{S}_{\tau}=\mathcal{S}_{\gamma, \tau} \oplus \mathcal{E}_{\tau} \oplus \mathcal{S}_{\gamma^{\prime}, \tau}$.

If $\mathcal{S}_{\tau}$ is separable, then any $\mathrm{G}^{1}$-spline function $\in \mathcal{S}_{\tau}$ can be uniquely decomposed as a sum of a $\mathrm{G}^{1}$-spline function $f$ with $T_{\gamma}^{\sigma_{0}}(f)=T_{\gamma}^{\sigma_{1}}(f)=T_{\gamma^{\prime}}^{\sigma_{0}}(f)=T_{\gamma^{\prime}}^{\sigma_{1}}(f)=0$, a function $g$ determined by its coefficients $T_{\gamma}^{\sigma_{0}}(g), T_{\gamma}^{\sigma_{1}}(g)$, and a function $h$ determined by its coefficients $T_{\gamma^{\prime}}^{\sigma_{0}}(h), T_{\gamma^{\prime}}^{\sigma_{1}}(h)$. This implies that there is no non-zero linear relation, induced by the $\mathrm{G}^{1}$ constraints, between the Bézier coefficients at the two vertices of an edge.

If $\mathcal{S}_{\tau}$ is not separable, there exists an element in $\mathcal{S}_{\tau}$ with non-zero Bézier coefficients at the two vertices, linearly independent of the $\mathrm{G}^{1}$-spline functions with zero coefficients at one of the vertices. If the mesh has more that one edge, this will induce the existence of $\mathrm{G}^{1}$-spline basis functions attached to vertices, whose support is not included in the neighborhood of cells adjacent to the vertex. Since we are interested in $\mathrm{G}^{1}$ spline spaces that admit a basis of functions with a local support, hereafter we only consider and construct separable $\mathrm{G}^{1}$-spline spaces.

We construct now explicit spaces of $\mathrm{G}^{1}$-spline functions along an edge. We consider b-spline spaces $\mathcal{R}_{d, \mathbf{t}}$ with a small degree $d(d=2, \ldots, 7)$ and a small number $m^{2}$ of control points per face $(m \leqslant 8)$. The analysis developed in this paper could be extended to a larger number $m$ of control points per edge, increasing the number of basis functions attached to an edge. 
The knots $\mathbf{t}$ of the b-spline functions are between 0 and 1 . The set of distinct knots is a uniform subdivision of the interval $[0,1]$, so that the b-spline functions share the same knots on the common edges. The glueing data on the edge are of the form $\mathfrak{a}(u)=a \Theta_{0}(u)-b \Theta_{1}(u), \mathfrak{b}(u)=-1$ with $a, b$ two parameters and $\Theta_{0}(u), \Theta_{1}(u)$ two functions interpolating 1 at 0 and 1 .

Translating the equations (2) and (3) into linear equations in the $4 m$ coefficients $c_{i, j}^{\sigma_{1}}(f), c_{j, i}^{\sigma_{0}}(f)$ for $0 \leqslant i \leqslant m-1$ and $0 \leqslant j \leqslant 1$, we compute bases of the spaces $\mathcal{S}_{\tau}, \mathcal{S}_{\gamma, \tau}, \mathcal{E}_{\tau}, \mathcal{S}_{\gamma^{\prime}, \tau}$ depending on the values of $a, b$ for a given edge $\tau$. This can be precomputed for given degree and knot vector of b-spline patches and for given type of glueing data (e.g. using a computer algebra system such as Maple).

\subsubsection{Basis of $\mathcal{E}_{\tau}$}

We compute a basis $e_{1}^{\tau}, \ldots, e_{l}^{\tau}$ of $\mathcal{E}_{\tau}$ defined by the equations $(2),(3), T_{\gamma}^{\tau}(f)=0$ and $T_{\gamma^{\prime}}^{\tau}(f)=0$. Notice that the functions $e_{i}^{\tau}$ are $\mathrm{G}^{1}$ splines on the whole topological space $\mathcal{M}$, since they are $\mathrm{G}^{1}$ along the edge $\tau$ and $T_{\gamma}^{\tau}\left(e_{i}^{\tau}\right)=T_{\gamma^{\prime}}^{\tau}\left(e_{i}^{\tau}\right)=0$. These will be called the edge basis functions of the edge $\tau$.

We denote by $\mathcal{B}_{\tau}^{1}=\left\{b_{i_{1}, j_{1}}^{\sigma_{k_{1}}}, \ldots, b_{i_{l}, j_{l}}^{\sigma_{k_{l}}}\right\}$ a set of free coefficients in the linear system of equations (2), (3), $T_{\gamma}^{\tau}(f)=0, T_{\gamma^{\prime}}^{\tau}(f)=0$. This is a Minimal Determining Set of coefficients for $\mathcal{E}_{\tau}$.

\subsubsection{Basis of $\mathcal{S}_{\gamma, \tau}$}

By definition of the space $\mathcal{S}_{\gamma, \tau}$, when the $\mathrm{G}^{1}$-spline space $\mathcal{S}_{\tau}$ is separable and ample, the map $T_{\gamma}^{\sigma}$ is injective on $\mathcal{S}_{\gamma, \tau}$ and its image is at least of dimension 4. This implies that the dimension of $\mathcal{S}_{\gamma, \tau}$ is at least 4. Since there are 3 independent relations between the 8 Taylor coefficients at a vertex $\gamma$ on the two faces $\sigma, \sigma^{\prime}$ (the coefficients on the common edge are equal and the derivatives along the edges adjacent to $\gamma$ are dependent), the dimension of $\mathcal{S}_{\gamma, \tau}$ is at most $8-3=5$.

For notational conveniency, we define $\delta(\gamma, \tau)=0$ if $\operatorname{dim}\left(\mathcal{S}_{\gamma, \tau}\right)=5$ and $\delta(\gamma, \tau)=1$ otherwise. If $\tau$ is a boundary edge, we let $\delta(\gamma, \tau)=0$. By definition $\operatorname{dim} S_{\gamma, \tau}=5-\delta(\gamma, \tau)$ We say that $\tau$ is a crossing edge at $\gamma$ if $\delta(\gamma, \tau)=1$ and a non-crossing edge otherwise. We define $\delta(\gamma)=\min \{\delta(\gamma, \tau) \mid \tau \ni \gamma\}$.

- If $\delta(\gamma, \tau)=1$ (crossing edge), $\operatorname{dim}\left(\mathcal{S}_{\gamma, \tau}\right)=4$ and a Minimal Determining Set of coefficients is associated to the b-spline functions $\mathcal{B}_{\gamma, \tau}^{0}=\left\{b_{0,0}^{\sigma_{0}}, b_{1,0}^{\sigma_{0}}, b_{0,1}^{\sigma_{0}}, b_{1,1}^{\sigma_{0}}\right\}$.

- If $\delta(\gamma, \tau)=0$ (non-crossing edge), $\operatorname{dim}\left(\mathcal{S}_{\gamma, \tau}\right)=5$ and a minimal determining set of $\mathcal{S}_{\gamma, \tau}$ is associated to the b-spline functions $\mathcal{B}_{\gamma, \tau}=\left\{b_{0,0}^{\sigma_{0}}, b_{1,0}^{\sigma_{0}}, b_{0,1}^{\sigma_{0}}, b_{1,1}^{\sigma_{0}}, b_{1,1}^{\sigma_{1}}\right\}$.

These sets are maximal sets of free coefficients in the linear system defining $\mathcal{S}_{\gamma, \tau}$. They are Minimal Determining Sets for $\mathcal{S}_{\gamma, \tau}$.

The space $\mathcal{S}_{\gamma, \tau}=\mathcal{S}_{\tau} \cap \mathcal{E}_{\tau}^{\perp} \cap \operatorname{ker} T_{\gamma^{\prime}}^{\tau}$ is defined by the equations (2), (3), $\left\langle f, e_{i}^{\tau}\right\rangle=0, i=1, \ldots, l$, and $T_{\gamma^{\prime}}^{\tau}(f)=0$.

As $\mathcal{B}_{\gamma, \tau}$ is a maximal set of free coefficients in this system, it can be transformed by linear combinations of these equations, into a system of the form

$$
\left[A_{\gamma, \tau} \mid \operatorname{Id}\right] \cdot c(f)=0
$$

where the columns of $A_{\gamma, \tau}$ are indexed by the coefficients $\mathcal{B}_{\gamma, \tau}$ and the last identity block indexed by the set $\mathcal{C}_{\gamma, \tau}$ of remaining coefficients among all coefficients of b-splines functions supported along $\tau$. The vector $c(f)$ is the vector of all the coefficients of functions supported along $\tau$.

Notice that this matrix $A_{\gamma, \tau}$ can be precomputed for each edge $\tau$, independently of the structure of the mesh. It depends only on the glueing data on $\tau$.

\subsubsection{Examples of ample separable spaces}

Hereafter, we describe cases of ample separable spaces of $\mathrm{G}^{1}$-splines for low $d$ and $m$. In these tables, we give the degree $d$, the knots $\mathbf{t}$, the number $m$ of control points along the edge, the glueing function $\mathfrak{a}(u)$ and the dimensions of $\mathcal{S}_{\tau}, \mathcal{S}_{\gamma, \tau}, \mathcal{E}_{\tau}, \mathcal{S}_{\gamma^{\prime}, \tau}$ for different values of $a$ and $b$. 
- $d=2, \mathbf{t}=\left[0^{3}, \frac{1}{4}, \frac{1}{2}, \frac{3}{4}, 1^{3}\right], m=6, \mathfrak{a}(u)=a(1-4 u) \mathbf{1}_{\left[0, \frac{1}{4}\right]}-b(4 u-3) \mathbf{1}_{\left[\frac{3}{4}, 1\right]}$

\begin{tabular}{|cc|cccc|}
\hline$a$ & $b$ & $\mathcal{S}_{\tau}$ & $S_{\gamma, \tau}$ & $\mathcal{E}_{\tau}$ & $\mathcal{S}_{\gamma^{\prime}, \tau}$ \\
\hline$\neq 0$ & $\neq 0$ & 10 & 4 & 2 & 4 \\
$=0$ & $\neq 0$ & 11 & 4 & 3 & 4 \\
$=0$ & $=0$ & 12 & 4 & 4 & 4 \\
\hline
\end{tabular}

This construction is closely related to the construction described in Reif (1995) with $\mathrm{C}^{1}$ biquadratic polynomials on each patch and the extraordinary vertices separated by 4 biquadratic patches.

A construction of $\mathrm{G}^{1}$-splines which are $\mathrm{C}^{1}$ bicubic b-splines on each patch with linear glueing data has been proposed in Hahmann et al. (2008). It applies under some genericity conditions on $\mathcal{M}$. Each face has $6 \times 6=36$ b-spline coefficients $(m=6)$. An explicit computation shows that the dimensions of $\mathcal{S}_{\tau}, S_{\gamma, \tau}, \mathcal{E}_{\tau}, \mathcal{S}_{\gamma^{\prime}, \tau}$ are respectively $11,4,2,4$ for $a \neq 0, b \neq 0$. Thus the space is not separable.

- $d=3, \mathbf{t}=\left[0^{4}, \frac{1}{3}, \frac{2}{3}, 1^{4}\right], m=6, \mathfrak{a}(u)=a(1-3 u) \mathbf{1}_{\left[0, \frac{1}{3}\right]}-b(3 u-2) \mathbf{1}_{\left[\frac{2}{3}, 1\right]}$.

\begin{tabular}{|cc|cccc|}
\hline$a$ & $b$ & $\mathcal{S}_{\tau}$ & $S_{\gamma, \tau}$ & $\mathcal{E}_{\tau}$ & $\mathcal{S}_{\gamma^{\prime}, \tau}$ \\
\hline$\neq 0$ & $=0$ & 10 & 4 & 2 & 4 \\
$=0$ & $=0$ & 12 & 4 & 4 & 4 \\
\hline
\end{tabular}

An explicit computation shows that when $a \neq 0, b \neq 0$, i.e. when none of the end points of the edge is a crossing vertex, the space $\mathcal{S}_{\tau}$ is not separable.

- $d=3, \mathbf{t}=\left[0^{4}, \frac{1}{3}, \frac{1}{3}, \frac{2}{3}, \frac{2}{3}, 1^{4}\right], m=8, \mathfrak{a}(u)=a(1-3 u) \mathbf{1}_{\left[0, \frac{1}{3}\right]}-b(3 u-2) \mathbf{1}_{\left[\frac{2}{3}, 1\right]}$ or $\mathfrak{a}(u)=a(3 u-$ $1)^{2} \mathbf{1}_{\left[0, \frac{1}{3}\right]}-b(3 u-2)^{2} \mathbf{1}_{\left[\frac{2}{3}, 1\right]}$.

\begin{tabular}{|cc|cccc|}
\hline$a$ & $b$ & $\mathcal{S}_{\tau}$ & $S_{\gamma, \tau}$ & $\mathcal{E}_{\tau}$ & $\mathcal{S}_{\gamma^{\prime}, \tau}$ \\
\hline$\neq 0$ & $\neq 0$ & 14 & 5 & 4 & 5 \\
$\neq 0$ & $=0$ & 15 & 5 & 6 & 4 \\
$=0$ & $=0$ & 16 & 4 & 8 & 4 \\
\hline
\end{tabular}

The case where $\mathfrak{a}$ is of degree 1 corresponds to the construction in Fan et al. (2008) and Peters et al. (2010), where the linear function $\mathfrak{a}$ is replaced by a piecewise linear function. In this case, the transition map is not necessarily $\mathrm{C}^{1}$.

The second case where $\mathfrak{a}$ is of degree 2 is a new construction. The glueing data $\mathfrak{a}$ is $\mathrm{C}^{1}$ for any value of $a$ and $b$.

- $d=3, \mathbf{t}=\left[0^{4}, \frac{1}{5}, \frac{2}{5}, \frac{3}{5}, \frac{4}{5}, 1^{4}\right], m=8, \mathfrak{a}(u)=a(1-5 u) \mathbf{1}_{\left[0, \frac{1}{5}\right]}-b(5 u-4) \mathbf{1}_{\left[\frac{4}{5}, 1\right]}$ or $\mathfrak{a}(u)=a(1-5 u)^{2} \mathbf{1}_{\left[0, \frac{1}{5}\right]}-$ $b(5 u-4)^{2} \mathbf{1}_{\left[\frac{4}{5}, 1\right]}$

\begin{tabular}{|cc|cccc|}
\hline$a$ & $b$ & $\mathcal{S}_{\tau}$ & $S_{\gamma, \tau}$ & $\mathcal{E}_{\tau}$ & $\mathcal{S}_{\gamma^{\prime}, \tau}$ \\
\hline$\neq 0$ & $\neq 0$ & 12 & 4 & 4 & 4 \\
$\neq 0$ & $=0$ & 14 & 4 & 6 & 4 \\
$=0$ & $=0$ & 16 & 4 & 8 & 4 \\
\hline
\end{tabular}

These two cases are also new constructions of $\mathrm{G}^{1}$-splines. The functions are $\mathrm{C}^{2}$ on each face and the glueing data is $\mathrm{C}^{1}$ for any value of $a$ and $b$ when $\mathfrak{a}$ is of degree 2 .

- $d=4, \mathbf{t}=\left[0^{5}, \frac{1}{2}, \frac{1}{2}, \frac{1}{2}, 1^{5}\right], m=8, \mathfrak{a}(u)=a(1-2 u)^{2} \mathbf{1}_{\left[0, \frac{1}{2}\right]}-b(2 u-1)^{2} \mathbf{1}_{\left[\frac{1}{2}, 1\right]}$

\begin{tabular}{|cc|cccc|}
\hline$a$ & $b$ & $\mathcal{S}_{\tau}$ & $S_{\gamma, \tau}$ & $\mathcal{E}_{\tau}$ & $\mathcal{S}_{\gamma^{\prime}, \tau}$ \\
\hline$\neq 0$ & $\neq 0$ & 14 & 5 & 4 & 5 \\
$\neq 0$ & $=0$ & 15 & 5 & 6 & 4 \\
$=0$ & $=0$ & 16 & 4 & 8 & 4 \\
\hline
\end{tabular}


This corresponds to the construction described in Bonneau et al. (2014) with $\mathrm{C}^{1}$ biquartic b-splines on each patch. It is also related to the construction in Peters (1995) where biquartic patches with quadratic transition maps are involved.

- $d=5, \mathbf{t}=\left[0^{6}, 1^{6}\right], m=6$,

For this degree, we consider glueing data of degree 1 when the vertices are not crossing vertices (i.e. $a \neq 0, b \neq 0): \mathfrak{a}(u)=a(1-u)-b u$.

\begin{tabular}{|cc|cccc|}
\hline$a$ & $b$ & $\mathcal{S}_{\tau}$ & $S_{\gamma, \tau}$ & $\mathcal{E}_{\tau}$ & $\mathcal{S}_{\gamma^{\prime}, \tau}$ \\
\hline$\neq 0$ & $\neq 0$ & 12 & 5 & 2 & 5 \\
\hline
\end{tabular}

When one of the vertices is a crossing vertex (i.e. $a=0$ or $b=0$, we use glueing data of degree 2 : $\mathfrak{a}(u)=a(1-u)^{2}$.

\begin{tabular}{|cc|cccc|}
\hline$a$ & $b$ & $\mathcal{S}_{\tau}$ & $S_{\gamma, \tau}$ & $\mathcal{E}_{\tau}$ & $\mathcal{S}_{\gamma^{\prime}, \tau}$ \\
\hline$\neq 0$ & $=0$ & 11 & 5 & 2 & 4 \\
$=0$ & $=0$ & 12 & 4 & 4 & 4 \\
\hline
\end{tabular}

This corresponds to the $\mathrm{G}^{1}$-space used for the IsoGeometric Analysis application in Section 5.

- $d=7, \mathbf{t}=\left[0^{8}, 1^{8}\right], m=8, \mathfrak{a}(u)=a(1-u)^{2}-b u^{2}$

\begin{tabular}{|cc|cccc|}
\hline$a$ & $b$ & $\mathcal{S}_{\tau}$ & $S_{\gamma, \tau}$ & $\mathcal{E}_{\tau}$ & $\mathcal{S}_{\gamma^{\prime}, \tau}$ \\
\hline$\neq 0$ & $\neq 0$ & 15 & 5 & 5 & 5 \\
$\neq 0$ & $=0$ & 15 & 5 & 6 & 4 \\
$=0$ & $=0$ & 16 & 4 & 8 & 4 \\
\hline
\end{tabular}

This is a new construction, which falls in the separable cases studied in Mourrain et al. (2016). The glueing data is $\mathrm{C}^{1}$ for any value of $a$ and $b$.

\section{2. $G^{1}$-splines around a vertex}

We present now a new method to construct $\mathrm{G}^{1}$-spline basis functions around a vertex $\gamma \in \mathcal{M}_{0}$ from the analysis of $\mathcal{S}_{\gamma, \tau}$ for $\tau \ni \gamma$, assuming $\mathcal{S}_{\tau}$ is ample and separable. Let $\sigma_{1}, \ldots, \sigma_{v}$ be the faces of $\mathcal{M}$ adjacent to the vertex $\gamma$, where $v$ is the valence of $\gamma$. We denote by $\mathcal{M}_{\gamma}$ the sub-topological surface induced by these faces and by $\mathfrak{g}_{\gamma}$ the corresponding glueing data. We assume that $\gamma$ is an interior point (the treatment of a boundary point will be similar). The edge between the faces $\sigma_{i}$ and $\sigma_{i+1}$ is $\tau_{i}=\left(\gamma, \gamma_{i}\right)$ for $i=1, \ldots, v$ (with the convention that $\left.\sigma_{v+1}=\sigma_{1}\right)$. The glueing data along the edge $\tau_{i}$ are denoted $\mathfrak{a}_{i}, \mathfrak{b}_{i}$. Let $\mathcal{S}_{\gamma} \subset \mathcal{S}\left(\mathcal{M}_{\gamma}, \mathfrak{g}_{\gamma}\right)$ be the space of $\mathrm{G}^{1}$-splines around the vertex $\gamma$, with support along the edges $\tau_{i}$ and with zero Taylor coefficients at the exterior vertices $\gamma_{i}$. Since the elements of $\mathcal{S}_{\gamma}$ have a support along the edges $\tau_{i}$ and zero Taylor coefficients at the exterior vertices $\gamma_{i}$, they define $\mathrm{G}^{1}$-splines on the global mesh: $S_{\gamma} \subset \mathcal{S}(\mathcal{M}, \mathfrak{g})$.

The space $\mathcal{E}_{\gamma} \subset \mathcal{S}_{\gamma}$ of $\mathrm{G}^{1}$-splines in $\mathcal{S}_{\gamma}$ supported along the edges $\tau_{i}$ with zero Taylor coefficients at $\gamma$ and at the exterior vertices $\gamma_{i}$ decomposes as $\mathcal{E}_{\gamma}=\oplus_{i=1}^{f} \mathcal{E}_{\tau_{i}}$ where $\mathcal{E}_{\tau_{i}}$ is the space of $\mathrm{G}^{1}$-splines defined in Section 3.1. Any element in $\mathcal{E}_{\gamma}$ is a sum of elements with support along the edges $\tau_{i}$ and zero Taylor coefficients at $\gamma$ and $\gamma_{i}$, that is an element of $\mathcal{E}_{\tau_{i}}$. A basis of $\mathcal{E}_{\tau_{i}}$ has been computed in Section 3.1.1.

The space $\mathcal{S}_{\gamma}$ decomposes as $\mathcal{S}_{\gamma}=\mathcal{E}_{\gamma} \oplus \mathcal{V}_{\gamma}$ where $\mathcal{V}_{\gamma}=\mathcal{E}_{\gamma}^{\perp} \cap \mathcal{S}_{\gamma}$ is the space orthogonal (and thus supplementary) to $\mathcal{E}_{\gamma}$ in $\mathcal{S}_{\gamma}$ (for the classical inner-product on their b-spline coefficients). We are going to construct a basis of $\mathcal{V}_{\gamma}$, that we will call the vertex basis functions of the vertex $\gamma$.

We assume for simplicity that either $v=4$ and $\delta\left(\gamma, \tau_{i}\right)=1$ for $i=1, \ldots, 4$ (crossing vertex) or $\delta\left(\gamma, \tau_{i}\right)=0$ for $i=1, \ldots, v$ (non-crossing vertex).

Vertex basis algorithm. Let $\gamma \in \mathcal{M}_{0}$ be a vertex with adjacent edges $\tau_{1}, \ldots, \tau_{v}$ and adjacent faces $\sigma_{1}, \ldots, \sigma_{v}$. 
- If $\delta\left(\gamma, \tau_{i}\right)=1$ (crossing vertex), then let

$$
G_{\gamma}=\left[b_{0,0}^{\sigma_{1}}, b_{1,0}^{\sigma_{1}}, b_{0,1}^{\sigma_{1}}, b_{1,1}^{\sigma_{1}}\right]
$$

be the coefficient matrix of the canonical basis elements $b_{0,0}^{\sigma_{1}}, b_{1,0}^{\sigma_{1}}, b_{0,1}^{\sigma_{1}}, b_{1,1}^{\sigma_{1}}$.

- If $\delta\left(\gamma, \tau_{i}\right)=0$, let

$$
G_{\gamma}=\left[b_{0,0}^{\sigma_{1}}, b_{1,0}^{\sigma_{1}}, b_{0,1}^{\sigma_{1}}, b_{1,1}^{\sigma_{1}}, \ldots, b_{1,1}^{\sigma_{v}}\right]
$$

be the coefficient matrix of the corresponding canonical basis elements.

For $i=1, \ldots, v$, we define the coefficients along the edge $\tau_{i}$ as follows

$$
G_{\gamma}\left[\mathcal{C}_{\tau_{i}},:\right]:=-A_{\gamma, \tau_{i}} G_{\gamma}\left[\mathcal{B}_{\tau_{i}},:\right]
$$

where $\mathcal{B}_{\tau_{i}}$ are the b-spline basis functions indexing the columns of $A_{\tau_{i}}$ and $\mathcal{C}_{\tau_{i}}$ are indexing the identity block in (8).

Proposition 3.2. The spline functions $G_{\gamma}$ constructed by this algorithm form a basis of $\mathcal{V}_{\gamma}$.

Proof. For each edge $\tau_{i}$, the restriction of the elements of $G_{\gamma}$ to $\mathcal{M}_{\tau_{i}}$ are in $\mathcal{S}_{\gamma, \tau_{i}}$ since, by construction, we have

$$
\left[A_{\gamma, \tau_{i}} \mid \text { Id }\right]\left[\begin{array}{l}
G_{\gamma}\left[\mathcal{B}_{\tau_{i}},:\right] \\
G_{\gamma}\left[\mathcal{C}_{\tau_{i}},:\right]
\end{array}\right]=\left[A_{\gamma, \tau_{i}} \mid \text { Id }\right]\left[\begin{array}{c}
G_{\gamma}\left[\mathcal{B}_{\tau_{i}},:\right] \\
-A_{\gamma, \tau_{i}} G_{\gamma}\left[\mathcal{B}_{\tau_{i}},:\right]
\end{array}\right]=0 .
$$

so that they satisfy the linear relations defining $\mathcal{S}_{\gamma, \tau_{i}}$. As this is true for all the edges $\tau_{i}$ containing $\gamma$, they are in $\mathcal{V}_{\gamma}$.

If $\delta\left(\gamma, \tau_{i}\right)=0$ (non-crossing vertex), the coefficients $c_{0,0}^{\sigma_{i}}, c_{1,0}^{\sigma_{i}}, c_{0,1}^{\sigma_{i}}, i=1, \ldots, v$ are linked by the relations $c_{0,0}^{\sigma_{i}}=c_{0,0}^{\sigma_{i+1}}, c_{0,1}^{\sigma_{i}}=c_{1,0}^{\sigma_{i+1}}$ and $c_{0,1}^{\sigma_{i+1}}-c_{0,0}^{\sigma_{i+1}}=\mathfrak{b}_{i}(0)\left(c_{1,0}^{\sigma_{i}}-c_{0,0}^{\sigma_{i}}\right)+\mathfrak{a}_{i}(0)\left(c_{0,1}^{\sigma_{i}}-c_{0,0}^{\sigma_{i}}\right)$. As the glueing data satisfies the cocyle condition 2.3, this system defines a linear space of dimension 3 . The coefficients $c_{1,1}^{\sigma_{1}}, \ldots, c_{1,1}^{\sigma_{v}}$ are free and the coefficients in $\mathcal{C}_{\tau_{i}}$ are determined by the relations (8). Thus the space $\mathcal{V}_{\gamma}$ defined by all these equations is of dimension $3+v$, which is also the number of elements in $G_{\gamma}$.

Let us show that the elements in $G_{\gamma}$ are linearly independent. By the linear transformation of the algorithm and the cocyle condition, the matrix $G_{\gamma}\left[\mathcal{B}_{\gamma},:\right]$ is not changed and is equal to the identity matrix. Thus the elements $G_{\gamma}$ are independent and, therefore, form a basis of $\mathcal{V}_{\gamma}$.

If $\delta\left(\gamma, \tau_{i}\right)=1$ (crossing vertex), a similar argument on the coefficients $c_{0,0}^{\sigma_{i}}, c_{1,0}^{\sigma_{i}}, c_{0,1}^{\sigma_{i}}, c_{1,1}^{\sigma_{i}}, i=1, \ldots, v$ and the cocyle condition 2.3 show that $\mathcal{V}_{\gamma}$ is of dimension 4 . Similarly, $G_{\gamma}\left[\mathcal{B}_{\gamma},:\right]$ is the identity matrix and $G_{\gamma}$ is a basis of $\mathcal{V}_{\gamma}$.

\subsection{Dimension formula for $\mathcal{S}_{d, \mathbf{t}}(\mathcal{M}, \mathfrak{g})$}

We consider here a degree $d$, a knot vector $\mathbf{t}$ which gives a separable and ample space of $\mathrm{G}^{1}$-splines $\mathcal{S}_{d, \mathbf{t}}(\mathcal{M}, \mathfrak{g})$.

Theorem 3.3. Assume that $\mathcal{S}_{d, \mathbf{t}}(\mathcal{M}, \mathfrak{g})$ is separable and ample then

$$
\operatorname{dim} \mathcal{S}_{d, \mathbf{t}}(\mathcal{M}, \mathfrak{g})=\left((m-4)^{2}+4\right) f_{2}+\sum_{\tau \in \mathcal{M}_{1}} \epsilon(\tau)-\sum_{(\gamma, \tau) \mid \gamma \in \tau} \delta(\gamma, \tau)+3 f_{0}+f_{0, \delta}
$$

where

- $f_{2}=\left|\mathcal{M}_{2}\right|$ is the number of faces of $\mathcal{M}$,

- $f_{0}=\left|\mathcal{M}_{0}\right|$ is the number of vertices of $\mathcal{M}$,

- $f_{0, \delta}$ is the number of vertices $\gamma \in \mathcal{M}$ such that $\delta(\gamma)=1$,

- $\epsilon(\tau)=\operatorname{dim}\left(\mathcal{E}_{\tau}\right)$, 
- $\delta(\gamma, \tau)=5-\operatorname{dim}\left(\mathcal{S}_{\gamma, \tau}\right)$ for an interior edge, $\delta(\gamma, \tau)=0$ for a boundary edge.

Proof. The dimension is obtained by counting the number of basis functions attached to faces, edges, and vertices using the construction of the previous section.

For each face $\sigma$, the b-spline basis function with interior control points are basis elements. There are $(m-4)^{2}$ such elements per face.

For each edge $\tau$, a basis of the space $\mathcal{E}_{\tau}$ are also basis elements of $\mathcal{S}_{\gamma, \tau}$.

For each vertex $\gamma$, the number of basis functions attached to it is $3+f_{\gamma}-\sum_{\tau \ni \gamma} \delta(\gamma, \tau)+\delta(\gamma)$, where $f_{\gamma}$ is the number of faces adjacent to $\gamma$. Since each face has 4 vertices, $\sum_{\gamma \in \mathcal{M}_{0}} f_{\gamma}=4 f_{2}$.

Let us check that these elements form a basis of the space $\mathcal{S}_{d, \mathbf{t}}(\mathcal{M})$. By construction, they are linearly independent. Given an element $g \in \mathcal{S}^{1}(\mathcal{M})$ let us prove that $g$ can be decomposed as a linear combination of the functions associated to the faces, edges and vertices. The elements associated to the faces are b-spline basis functions. By construction, the elements associated to a vertex $\gamma$ have one of their b-spline coefficients equal to 1 and the other coefficients indexed by $\mathcal{B}_{\gamma}$ equal to zero. By subtracting a linear combination of these elements, we can assume that the coefficients of $g$ indexed by $\mathcal{B}_{\gamma}$ for $\gamma \in \mathcal{M}_{0}$ or interior to a face $\sigma$ vanish. The $G^{1}$ constraints induced by the relations (3) at $u_{1}=0$ imply that $T_{\gamma}^{\tau}(g)=0$ for all $\gamma \in \mathcal{M}_{0}$ and $\tau \in \mathcal{M}_{1}$ s.t. $\gamma \in \tau$. Therefore, $g$ decomposes as

$$
g=\sum_{\tau \in \mathcal{M}_{1}} g_{\tau}
$$

where $g_{\tau} \in \mathcal{E}_{\tau}$ is a linear combination of the basis elements associated to the edge $\tau$. Consequently, $g$ belongs to the vector space spanned by the elements associated to the faces, edges and vertices.

Summing up all these terms gives formula (9) for the dimension of $\mathcal{S}_{d, \mathbf{t}}(\mathcal{M})$.

\subsection{Example}

We consider the knot vector $\mathbf{t}=\left[0^{4}, \frac{1}{2}, \frac{1}{2}, 1^{4}\right]$ defining bicubic $C^{1}$ splines with $m=6$ control points per edge. We take glueing data of the form $\mathfrak{a}(u)=a(1-3 u) \mathbf{1}_{\left[0, \frac{1}{3}\right]}-b(3 u-2) \mathbf{1}_{\left[\frac{2}{3}, 1\right]}$. Let $\tau$ be an interior edge of $\mathcal{M}$ and let $\sigma_{0}, \sigma_{1}$ be the adjacent faces to $\tau$. We have the separability property for $b=0$ :

\begin{tabular}{|cc|cccc|}
\hline$a$ & $b$ & $\mathcal{S}_{\tau}$ & $S_{\gamma, \tau}$ & $\mathcal{E}_{\tau}$ & $\mathcal{S}_{\gamma^{\prime}, \tau}$ \\
\hline$\neq 0$ & $=0$ & 11 & 5 & 2 & 4 \\
$=0$ & $=0$ & 12 & 4 & 4 & 4 \\
\hline
\end{tabular}

The computation of a basis of $\mathcal{E}_{\tau}$ for $a \neq 0, b=0$ yields

$$
\mathcal{B}_{\tau}=\left[-b_{1,2}^{\sigma_{0}}+b_{2,1}^{\sigma_{1}},-b_{1,3}^{\sigma_{0}}+b_{3,1}^{\sigma_{1}}\right]
$$

The relations defining $\mathcal{S}_{\gamma, \tau}$ are of the form

$$
\left[\begin{array}{c}
c_{0,0}^{\sigma_{1}} \\
c_{1,0}^{\sigma_{1}} \\
c_{0,1}^{\sigma_{1}} \\
c_{0,2}^{\sigma_{0}} \\
c_{0,3}^{\sigma_{0}} \\
c_{1,2}^{\sigma_{0}} \\
c_{1,3}^{\sigma_{0}} \\
c_{2,0}^{\sigma_{1}} \\
c_{2,1}^{\sigma_{1}} \\
c_{3,0}^{\sigma_{1}} \\
c_{3,1}^{\sigma_{1}}
\end{array}\right]=\left[\begin{array}{ccccc}
1 & 0 & 0 & 0 & 0 \\
0 & 1 & 0 & 0 & 0 \\
2-a & a & -1 & 0 & 0 \\
0 & \frac{a-3}{a} & 0 & \frac{3}{2 a} & \frac{3}{2 a} \\
0 & \frac{a-3}{a} & 0 & \frac{3}{2 a} & \frac{3}{2 a} \\
0 & \frac{a-3}{a} & 0 & \frac{3}{2 a} & \frac{3}{2 a} \\
0 & \frac{a-3}{a} & 0 & \frac{3}{2 a} & \frac{3}{2 a} \\
0 & \frac{a-3}{a} & 0 & \frac{3}{2 a} & \frac{3}{2 a} \\
0 & \frac{a-3}{a} & 0 & \frac{3}{2 a} & \frac{3}{2 a} \\
0 & \frac{a-3}{a} & 0 & \frac{3}{2 a} & \frac{3}{2 a} \\
0 & \frac{a-3}{a} & 0 & \frac{3}{2 a} & \frac{3}{2 a}
\end{array}\right]\left[\begin{array}{c}
c_{0,0}^{\sigma_{0}} \\
c_{0,1}^{\sigma_{0}} \\
c_{1,0}^{\sigma_{0}} \\
c_{1,1}^{\sigma_{0}} \\
c_{1,1}^{\sigma_{1}}
\end{array}\right]
$$


where $-A_{\gamma, \tau}$ is the matrix appearing in this system.

For a vertex $\gamma$ of valence 3 (with $a=2 \cos \left(\frac{2 \pi}{3}\right)=-1$ ) adjacent to the faces $\sigma_{0}, \sigma_{1}, \sigma_{2}$, the 6 spline basis functions of $\mathcal{S}_{\gamma}$ are:

$$
\begin{aligned}
& b_{0,0}^{\sigma_{0}}+b_{0,0}^{\sigma_{2}}+3 b_{1,0}^{\sigma_{2}}+24 b_{2,1}^{\sigma_{2}}+12 b_{2,0}^{\sigma_{2}}+12 b_{3,0}^{\sigma_{2}}+b_{0,0}^{\sigma_{1}}+3 b_{0,1}^{\sigma_{1}}+12 b_{0,2}^{\sigma_{1}}+12 b_{0,3}^{\sigma_{1}}+24 b_{1,3}^{\sigma_{1}}, \\
& b_{0,1}^{\sigma_{0}}+4 b_{0,2}^{\sigma_{0}}+4 b_{0,3}^{\sigma_{0}}+8 b_{1,3}^{\sigma_{0}}-b_{1,0}^{\sigma_{2}}-8 b_{2,1}^{\sigma_{2}}-4 b_{2,0}^{\sigma_{2}}-4 b_{3,0}^{\sigma_{2}}+b_{1,0}^{\sigma_{1}}+4 b_{2,0}^{\sigma_{1}}+4 b_{3,0}^{\sigma_{1}}-b_{0,1}^{\sigma_{1}}+8 b_{2,1}^{\sigma_{1}} \\
& \quad-4 b_{0,2}^{\sigma_{1}}-4 b_{0,3}^{\sigma_{1}}-8 b_{1,3}^{\sigma_{1}}, \\
& b_{1,0}^{\sigma_{0}}+4 b_{2,0}^{\sigma_{0}}+4 b_{3,0}^{\sigma_{0}}+8 b_{2,1}^{\sigma_{0}}+4 b_{0,3}^{\sigma_{2}}+4 b_{0,2}^{\sigma_{2}}+b_{0,1}^{\sigma_{2}}+8 b_{1,3}^{\sigma_{2}}-b_{1,0}^{\sigma_{2}}-8 b_{2,1}^{\sigma_{2}}-4 b_{2,0}^{\sigma_{2}}-4 b_{3,0}^{\sigma_{2}}-b_{0,1}^{\sigma_{1}} \\
& \quad-4 b_{0,2}^{\sigma_{1}}-4 b_{0,3}^{\sigma_{1}}-8 b_{1,3}^{\sigma_{1}}, \\
& -2 b_{2,0}^{\sigma_{0}}-2 b_{3,0}^{\sigma_{0}}+b_{1,1}^{\sigma_{0}}-3 b_{2,1}^{\sigma_{0}}-2 b_{0,2}^{\sigma_{0}}-2 b_{0,3}^{\sigma_{0}}-3 b_{1,3}^{\sigma_{0}}-2 b_{0,3}^{\sigma_{2}}-2 b_{0,2}^{\sigma_{2}}-3 b_{1,3}^{\sigma_{2}}-2 b_{2,0}^{\sigma_{1}}-2 b_{3,0}^{\sigma_{1}}-3 b_{2,1}^{\sigma_{1}}, \\
& -2 b_{0,2}^{\sigma_{0}}-2 b_{0,3}^{\sigma_{0}}-3 b_{1,3}^{\sigma_{0}}-3 b_{2,1}^{\sigma_{2}}-2 b_{2,0}^{\sigma_{2}}-2 b_{3,0}^{\sigma_{2}}-2 b_{2,0}^{\sigma_{1}}-2 b_{3,0}^{\sigma_{1}}+b_{1,1}^{\sigma_{1}}-3 b_{2,1}^{\sigma_{1}}-2 b_{0,2}^{\sigma_{1}}-2 b_{0,3}^{\sigma_{1}}-3 b_{1,3}^{\sigma_{1}}, \\
& -2 b_{2,0}^{\sigma_{0}}-2 b_{3,0}^{\sigma_{0}}-3 b_{2,1}^{\sigma_{0}}-2 b_{0,3}^{\sigma_{2}}-2 b_{0,2}^{\sigma_{2}}-3 b_{1,3}^{\sigma_{2}}+b_{1,1}^{\sigma_{2}}-3 b_{2,1}^{\sigma_{2}}-2 b_{2,0}^{\sigma_{2}}-2 b_{3,0}^{\sigma_{2}}-2 b_{0,2}^{\sigma_{1}}-2 b_{0,3}^{\sigma_{1}}-3 b_{1,3}^{\sigma_{1}} .
\end{aligned}
$$

\section{Application to point cloud fitting}

Let $\mathcal{P}=\left\{p_{1}, \ldots, p_{n}\right\}$ be a cloud of points in $\mathbb{R}^{3}$ and $\mathcal{N}=\left\{v_{1}, \ldots, v_{n}\right\}$ their corresponding normals, representing a smooth surface that has the same topology as the topological complex $\mathcal{M}$. The goal of this section is to produce a smooth surface that is as close as possible to the cloud of points using the $G^{1}$-basis constructed from $\mathcal{M}$.

Denote by $\left(g_{i}\right)_{i \in I}, I=\{1, . ., r\}, r \in \mathbb{N}$ the finite basis of the space $\mathcal{S}_{d, t}(\mathcal{M}, \mathfrak{g})$ of $G^{1}$ splines over $\mathcal{M}$ of degree $d$ and with knots sequence $t$. The functions $g_{i}$ are used to parametrise $3 \mathrm{~d}$-surfaces, by taking linear combinations:

$$
\mathfrak{s}=\sum_{i \in I} \overline{\mathbf{s}}_{i} g_{i}
$$

with coefficients $\overline{\mathbf{s}}_{i} \in \mathbb{R}^{3}$ for $i \in I$.

Over each face $\sigma$ of the mesh $\mathcal{M}$, the functions $g_{i}$ are represented as linear combinations of the b-spline basis function with coefficients that we denote $c_{k, l}^{\sigma}(\mathfrak{s})$. Hereafter, we will also use $g_{i}$ to denote the vector of all coefficients $c_{k, l}^{\sigma}\left(g_{i}\right)$ for all faces $\sigma \in \mathcal{M}_{2}$ and $\bar{G}=\left[g_{i}\right]_{i \in I}$ the matrix, which columns are the vectors $g_{i}$. The $N \times 3$ matrix $\bar{C}=\left[c_{k, l}^{\sigma}(\mathfrak{s})\right]$ which rows are the b-spline coefficients $c_{k, l}^{\sigma}(\mathfrak{s})$ of the surface $h$ will be written by means of the $l \times 3$ matrix $\overline{\mathbf{s}}$ which rows are the points $\overline{\mathbf{s}}_{i}$ :

$$
\bar{C}=\bar{G}\left(\begin{array}{c}
\overline{\mathbf{s}}_{1} \\
\vdots \\
\overline{\mathbf{s}}_{l}
\end{array}\right)=\bar{G} \overline{\mathbf{s}}
$$

For simplicity, we will use the following notation:

$$
\mathbf{s}=\left(\begin{array}{l}
\overline{\mathbf{s}}[:, 1] \\
\overline{\mathbf{s}}[:, 2] \\
\overline{\mathbf{s}}[:, 3]
\end{array}\right), \quad C=\left(\begin{array}{l}
\bar{C}[:, 1] \\
\bar{C}[:, 2] \\
\bar{C}[:, 3]
\end{array}\right), \quad G=\operatorname{diag}(\bar{G}, \bar{G}, \bar{G}),
$$

where, for a matrix $M, M[:, i]$ indicates the $i^{t h}$ column of $M$. With this notation, we have $C=G \mathbf{s}$.

In order to obtain the most accurate representation of $\mathcal{P}$ by $G^{1}$ splines, we compute $\mathbf{s}$ by minimizing a weighted combination of square distance and fairing energies. We recall briefly these standard energy terms (see e.g. Greiner et al. (1994); Wang et al. (2006)) and give their matrix formulation in terms of the coefficients in the $G^{1}$ basis.

Distance energy. Given a (uniform) distribution $\mathcal{U}=\left\{u_{1}, \ldots, u_{n}\right\}$ of parameters in $\mathcal{M}$ and a set $\mathcal{P}$ of points (with normals), we use either 
- the classical point-wise square distance energy

$$
E_{P}(\mathbf{s})=\sum_{u \in \mathcal{U}}\left\|p_{u}-\mathfrak{s}(u)\right\|^{2}
$$

where the pairing between the parameters $u \in \mathcal{U}$ and points $p_{u} \in \mathcal{P}$ is obtained from an initial parameterisation $\mathfrak{s}_{0}$, by associating to $u \in \mathcal{U}$ the closest point $p_{u} \in \mathcal{P}$ to $\mathfrak{s}_{0}(u$ ) (we use a kd tree algorithm to compute closest points).

- or the square distance between the planes at the points $p_{i} \in \mathcal{P}$ with normal $v_{i} \in \mathcal{N}$ and the points $\mathfrak{s}\left(u_{i}\right)$ is:

$$
E_{T}(\mathbf{s})=\sum_{i=1, . ., l}\left[v_{i} \cdot\left(p_{i}-\mathfrak{s}\left(u_{i}\right)\right)^{t}\right]^{2}
$$

for $\mathbf{s} \in \mathcal{S}(\mathcal{M}, \mathfrak{g})$.

Other distance minimizations can be used, such as the so-called Squared Distance Minimization Wang et al. (2006), which involves the principal curvatures.

Fairing energy. To reduce oscillations in the computed surface, we use a regularization term (see e.g. Greiner et al. (1994))

$$
\mathcal{F}_{k}(g)=\int_{0}^{1} \int_{0}^{1}\left(\partial_{s}^{k} g(s, t)\right)^{2}+\left(\partial_{t}^{k} g(s, t)\right)^{2} d s d t
$$

In the experimentation, we use the regularization terms $\mathcal{F}_{1}$ and $\mathcal{F}_{2}$. To avoid an explicit computation of the integrals, we further simplify them into the following expressions involving directly the b-spline coefficients:

$$
\tilde{\mathcal{F}}_{1}(g)=\sum_{0 \leqslant i, j \leqslant n-1}\left\|\left(\Delta_{1} c\right)_{i, j}\right\|^{2}+\left\|\left(\Delta_{2} c\right)_{i, j}\right\|^{2}, \quad \tilde{\mathcal{F}}_{2}(g)=\sum_{1 \leqslant i, j \leqslant n-1}\left\|\left(\Delta_{1}^{2} c\right)_{i, j}\right\|^{2}+\left\|\left(\Delta_{2}^{2} c\right)_{i, j}\right\|^{2}
$$

with $\left(\Delta_{1} c\right)_{i, j}=c_{i+1, j}-c_{i, j}$, and $\left(\Delta_{2} c\right)_{i, j}=c_{i, j+1}-c_{i, j},\left(\Delta_{1}^{2} c\right)_{i, j}=c_{i, j}-\frac{\left(c_{i+1, j}+c_{i-1, j}\right)}{2}$ and $\left(\Delta_{2}^{2} c\right)_{i, j}=$ $c_{i, j}-\frac{\left(c_{i, j+1}+c_{i, j-1}\right)}{2}$. As the b-spline coefficients $C$ of $h$ are such that $C=G \mathbf{s}$, these energy terms are of the form $\mathbf{s}^{T} G^{T} A_{i} G \mathbf{s}$ where $A_{i}$ is the coefficient matrix of $\tilde{\mathcal{F}}_{i}$ in the b-spline basis (for $i=1,2$ ).

The final formula that we minimize is of the form:

$$
T_{\text {tot }}(\mathbf{s})=w_{1} E_{P}(\mathbf{s})+w_{2} E_{T}(\mathbf{s})+w_{3} \tilde{\mathcal{F}}_{1}(G \mathbf{s})+w_{4} \tilde{\mathcal{F}}_{2}(G \mathbf{s})
$$

where $w_{i}$ are weights, which are chosen manually depending on the type of the point cloud; the more the point cloud is noisy, the larger the fairing energy weights must be. The total energy $T_{t o t}(\mathbf{s})$ is a quadratic function of $\mathbf{s}$, and its minimum(s) can be obtained by solving $\nabla T_{t o t}(\mathbf{s})=0$, leading to the following linear system

$$
\left(w_{1} D^{T} D+w_{2} \tilde{D}^{T} \tilde{D}+w_{3} G^{T} A_{1} G+w_{4} G^{T} A_{2} G\right) \mathbf{s}-w_{1} \tilde{P}^{T} \tilde{D}-w_{2} P^{T} D=0
$$

\subsection{Illustrations}

As we said in previous sections the basis functions $\left(g_{i}\right)_{i \in I}$ are precomputed. They are represented by the sparse line vectors of $G$, this yields sparse matrices $D, \tilde{D}$ and vectors $P, \tilde{P}$. The matrices $A_{i}$ for $i=1,2$ are diagonal by blocks of size at most 16 , this can be proved by a combinatorial argument. This implies in particular that the total system is sparse.

We present in Fig. 1 and 2 some results of fitting surfaces, the computations were made with the Julia programming language, the visualization is done with the software $\mathrm{Axl}^{1}$.

The cloud of points in Fig. 1(c-e) and first row of Fig. 2 is taken from a smooth surface, made by building a scaffolding of a skeleton from Fuentes et al. (2017), then by applying a Catmull-Clark subdivision algorithm for smoothing.

${ }^{1}$ axl.inria.fr 

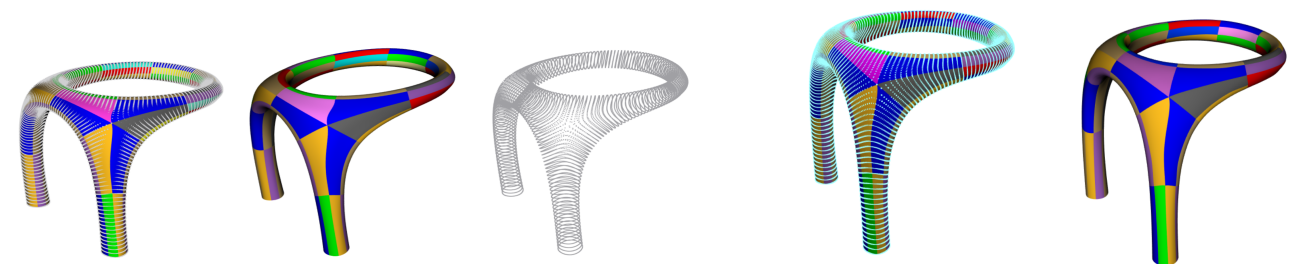

(a) Degree bi-5 Bézier patches with knot vector $\mathbf{t}=\left[0^{6}, 1^{6}\right]$ and quadratic glueing data. $F=96$,

(b) Degree bi-3 bsplines patches with knots $N=1180, P=24702, e_{\text {ave }}=0.0029685196$, $e_{\max }=0.00989258$. vector $\mathbf{t}=\left[0^{4}, \frac{1}{3}, \frac{2}{3}, 1^{4}\right]$ and linear glueing data. cloud of points as in (a). $F=96, N=1180$,

$$
\begin{gathered}
P=24702, e_{\text {ave }}=0.0015991, \\
e_{\max }=0.01391856 .
\end{gathered}
$$
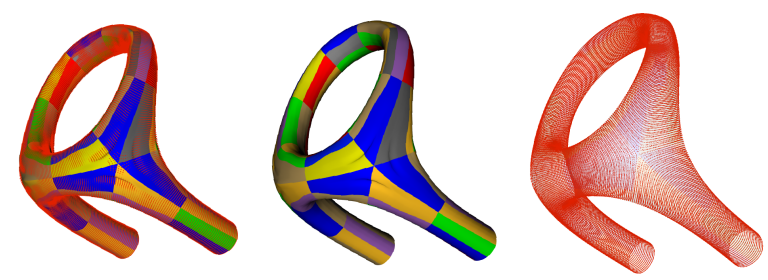

(c) Degree bi-3 bsplines patches with knots vector $\left[0^{4},(1 / 3)^{2},(2 / 3)^{2}, 1^{4}\right]$. This fitting exhibits a quality defect along the knots line that are close to singular vertices, because the element patches are $C^{1} . F=96, N=1980$, $P=24702, e_{a v e}=0.00594, e_{\max }=0.050988$.

Figure 1: Comparison of fitting of 3d-geometric objects with different basis. The first column is for the cloud of points and the fitting smooth surface, the second one for the fitting surfaces, and the last one is for the cloud of points alone. $F$ is the number of faces of the quad mesh, $N$ is the dimension of the $\mathrm{G}^{1}$ space, $P$ is the number of points, $e_{a v e}, e_{\max }$ are respectively the relative average error $\sum_{u \in \mathcal{U}} \frac{\left\|p_{u}-\mathfrak{s}(u)\right\|}{P \Delta}$ and relative maximum error $\max _{u \in \mathcal{U}} \frac{\left\|p_{u}-\mathfrak{s}(u)\right\|}{\Delta}$ where $\Delta$ is the diameter of the point cloud. 

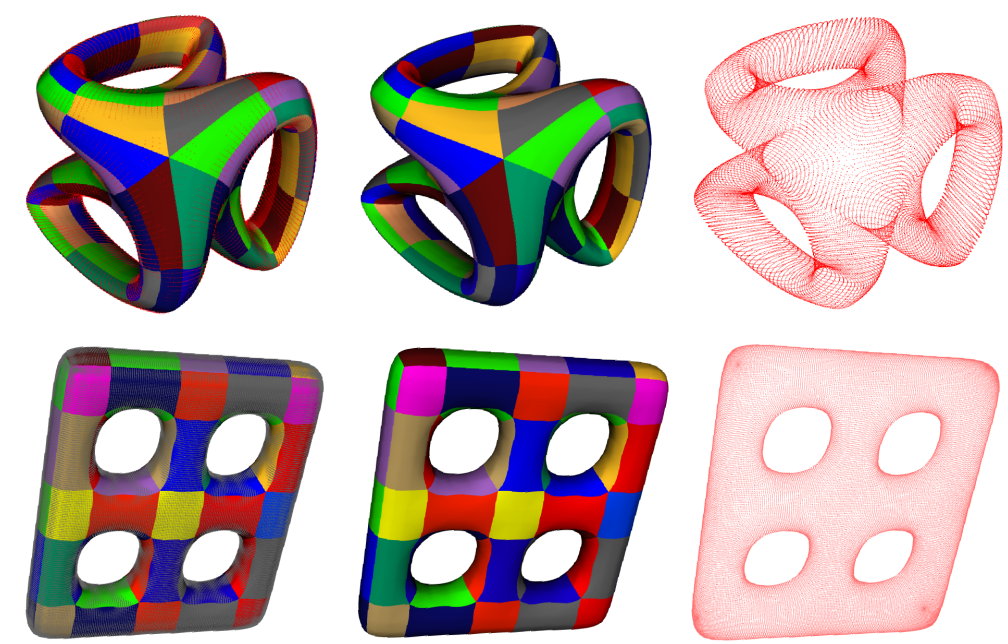

Figure 2: Fitting of 3d-geometric objects. The first column is for the cloud of points and the fitting smooth surface, the second one for the fitting surfaces, and the last one is for the cloud of points alone. We use the same b-spline type as in Fig. 1(e), with $F=144, N=2808, P=36860, e_{a v e}=0.00748197, e_{\max }=0.0324516$ for the first line example.

\section{Application in isogeometric analysis}

In this section, the proposed geometrically smooth spline bases will be applied in isogeometric analysis with complex geometry.

\subsection{Model problem and technique details}

Consider the following two-dimensional heat diffusion example as an illustrative model problem:

$$
\begin{aligned}
-\Delta T(\boldsymbol{x}) & =f(\boldsymbol{x}) & & \text { in } \Omega \subset \mathbb{R}^{2} \\
T(\boldsymbol{x}) & =0 & & \text { on } \partial \Omega_{D}
\end{aligned}
$$

where $\Delta$ is the Laplacian operator, $\Omega$ is the computational domain parameterized by the proposed geometrically smooth spline bases, $T(\boldsymbol{x})$ is the unknown heat field, and $f(\boldsymbol{x})$ is the heat source function. The trial and test spaces are defined as:

$$
\begin{aligned}
& \mathcal{U}=\left\{T \in H^{1}(\Omega): T=T_{D} \text { on } \partial \Omega\right\} \\
& \mathcal{V}=\left\{\psi \in H^{1}(\Omega): \psi=0 \text { on } \partial \Omega\right\} .
\end{aligned}
$$

The variational problem can be stated as: find the solution $T^{h} \in \mathcal{U}^{h} \subset \mathcal{U}$ such that:

$$
\int_{\Omega} \nabla T^{h}(\boldsymbol{x}) \cdot \nabla \psi^{h}(\boldsymbol{x}) d \Omega=\int_{\Omega} f(\boldsymbol{x}) \cdot \psi^{h}(\boldsymbol{x}) d \Omega \quad \forall \psi^{h} \in \mathcal{V}^{h} \subset \mathcal{V} .
$$

which can be written as

$$
a\left(T^{h}, \psi^{h}\right)=\left\langle f, \psi^{h}\right\rangle \quad \forall \psi^{h} \in \mathcal{V}^{h},
$$


where

$$
\begin{aligned}
a\left(T^{h}, \psi^{h}\right) & =\int_{\Omega} \nabla T^{h}(\boldsymbol{x}) \cdot \nabla \psi^{h}(\boldsymbol{x}) d \Omega, \\
\left\langle f, \psi^{h}\right\rangle & =\int_{\Omega} f(\boldsymbol{x}) \cdot \psi^{h}(\boldsymbol{x}) d \Omega .
\end{aligned}
$$

In the isogeometric analysis framework, the solution field $T^{h}$ will be represented by the proposed geometrically smooth spline bases, that is,

$$
T^{h}=\sum_{i=1}^{N} g_{i}(\mathbf{u}) T_{i},
$$

where $T_{i}$ are unknown variables to be solved, $g_{i}(\mathbf{u})$ are geometrically smooth spline basis functions defined on each face $\sigma$ from its b-spline coefficients $c_{k, l}^{\sigma}\left(g_{i}\right), \mathbf{u}^{\sigma}=\left(\xi^{\sigma}, \eta^{\sigma}\right)$ are the domain parameters associated to the face $\sigma$ of the parametric domain $\mathcal{P}, N$ is the number of basis functions. The test function $\psi^{h}$ is also defined as follows :

$$
\psi^{h}=g_{i}(\mathbf{u}) .
$$

Then a linear system can be obtained from Eq. (16),

$$
\boldsymbol{A T}=\boldsymbol{b}
$$

in which $\boldsymbol{T}=\left[T_{i}\right]$ are unknown variables. The entries in stiffness matrix $\boldsymbol{A}=\left[A_{i, k}\right]$ and right-hand side $\boldsymbol{b}=\left[b_{i}\right]$ can be computed as follows,

$$
\begin{aligned}
A_{i, k} & =\int_{\mathcal{P}} \nabla_{\mathbf{u}} g_{k}(\mathbf{u}) B(\mathbf{u})^{T} B(\mathbf{u}) \nabla_{\mathbf{u}} g_{k}(\mathbf{u}) J(\mathbf{u}) d \mathcal{P} \\
b_{i} & =\int_{\mathcal{P}} f(\mathcal{F}(\mathbf{u})) \cdot g_{k}(\mathbf{u}) J(\mathbf{u}) d \mathcal{P} .
\end{aligned}
$$

where $\mathcal{F}(\mathbf{u})=(x(\xi, \eta), y(\xi, \eta))$ is defined as in Eq. (10), $J(\mathbf{u})$ is the Jacobian of the transformation,

$$
J(\mathbf{u})=\left|\begin{array}{ll}
x_{\xi} & y_{\xi} \\
x_{\eta} & y_{\eta}
\end{array}\right|,
$$

$B(\mathbf{u})$ is the transposed of the inverse of the Jacobian matrix.

\subsection{Examples}

In this subsection, a numerical example is presented to demonstrate the effectiveness of the proposed simulation method with geometrically smooth spline bases.

We consider a heat diffusion problem with the following exact solution

$$
T(\boldsymbol{x})=10 \sin \left(\frac{\pi}{30}(x+y+30)\right) \sin \left(\frac{\pi}{30}(x+y-30)\right) \sin \left(\frac{\pi}{30}(x-y-30)\right) \sin \left(\frac{\pi}{30}(x-y+30)\right) .
$$

The computational domain is a square $[-30,30] \times[-30,30]$, which is parameterized by a quintic $G^{1}$ spline. The parametric mesh is shown in Fig. 3(a), and the corresponding parameterization with 52 patches is presented in Fig 3(b) and (c). The corresponding IGA numerical solution is shown in Fig. 3(d) and (e), and the corresponding error colormap is shown in Fig. 3(f). We observe that the proposed IGA framework based on geometrically smooth splines achieves an accuracy similar to the one using $C^{0}$ multi-patch method.

It should be mentioned that the IGA solution surface is also $G^{1}$ according to the property of geometrically smooth splines. The $C^{1}$ regularity is not required in the discretization of the heat diffusion problem. However, the solution of this problem is $C^{1}$ and the experiment shows that the capacity of the $\mathrm{G}^{1}$-spline functions to approximate such a solution. Moreover, since their dimension is smaller than the corresponding $\mathrm{G}^{0}$ spline space dimension, the induced linear algebra computation is also less costly. 


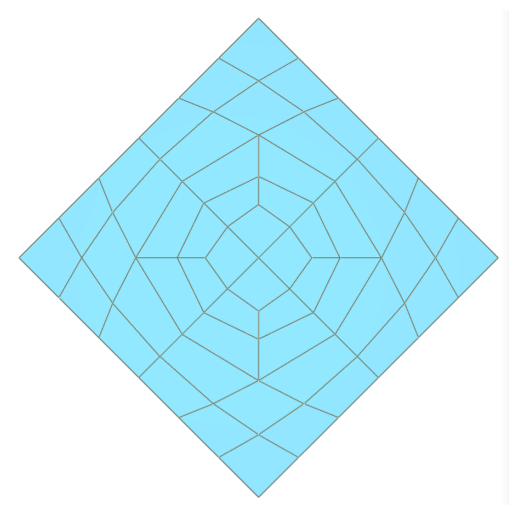

(a) parametric mesh

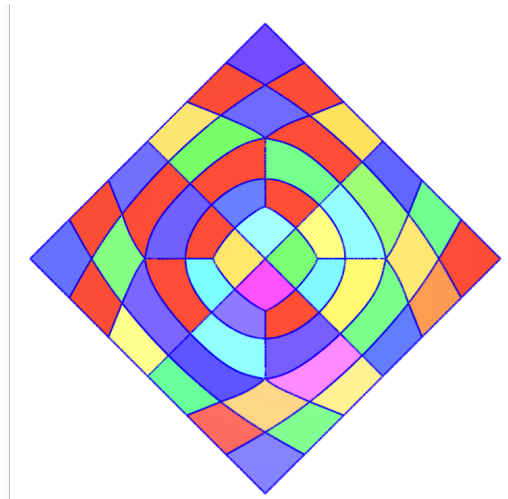

(b) parameterization with 52 Bézier patches

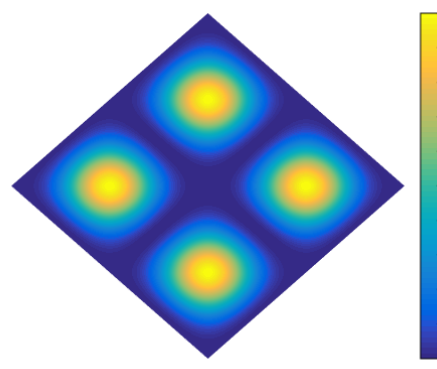

(e) IGA solution with colormap

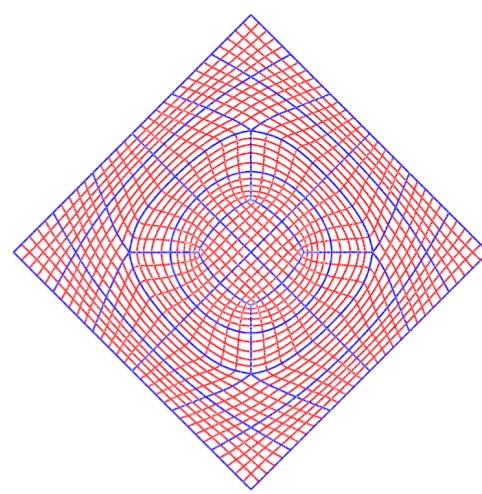

(c) parameterization with iso-parametric curves

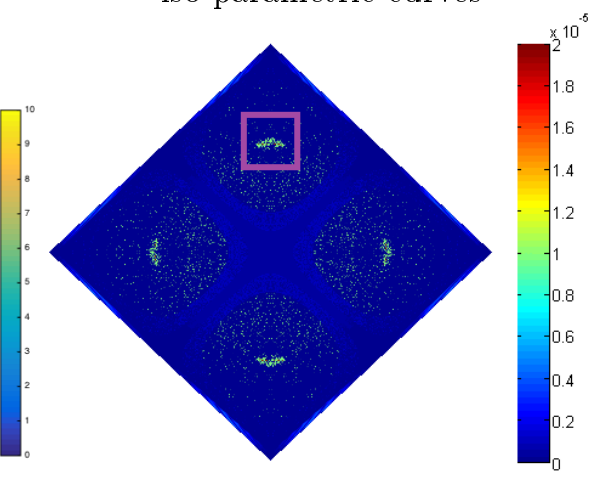

(f) Level 3 error colormap

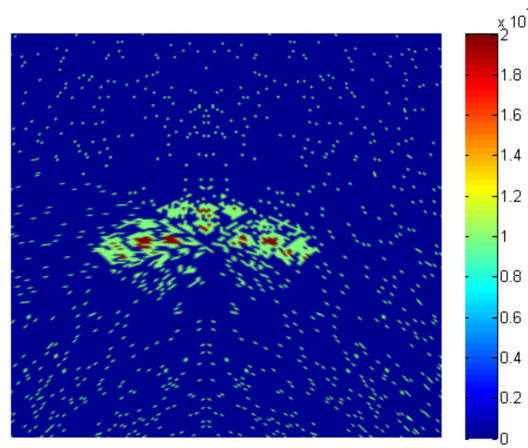

(f) Zoom into the level 3 error colormap

Figure 3: Numerical example of IGA with geometrically smooth splines.

\begin{tabular}{|c|c|c|c|c|}
\hline Level of subdivision & 0 & 1 & 2 & 3 \\
\hline Dimension & 529 & 1993 & 7729 & 30433 \\
\hline$L^{2}$ Error & 0.00215138 & 0.000427664 & $3.14061 \mathrm{e}-005$ & $2.90809 \mathrm{e}-006$ \\
\hline Rate of convergence & - & 2.33 & 3.77 & 3.43 \\
\hline
\end{tabular}

Figure 4: Estimated rate of convergence 


\section{Conclusion and future work}

We have presented a new method for efficient construction of $G^{1}$ spline basis on quadrangular meshes of arbitrary topology. We provide details about the degrees of freedom that we obtain depending on different kind of b-spline patches, and glueing data. Among the results, we have tested different bi-3 and bi- $5 G^{1}$ basis constructions for fitting point clouds, and we have tested bi-5 $G^{1}$ splines to solve a heat diffusion problem by an isogeometric finite element method.

In future works, we aim at analyzing the convergence rate of the approximation error when we perform mesh refinements and at combining fitting techniques for domain parametrizations with isogeometric analysis methods to reduce the approximate error.

Acknowledgements: The work of A. Blidia and B. Mourrain is partially supported by the European Union's Horizon 2020 research and innovation programme under the Marie Sklodowska-Curie grant agreement $\mathrm{N}^{\circ} 675789$ (ARCADES).

The research of Gang Xu was supported by the National Nature Science Foundation of China under grant nos. 61761136010, 61772163, the NSFC-Zhejiang Joint Fund for the Integration of Industrialization and Informatization Grant No. (U1909210), and the Science Challenge Project with No. TZZT2016002.

\section{Bibliography}

Peter Alfeld. Bivariate spline spaces and minimal determining sets. Journal of Computational and Applied Mathematics, 119(1-2):13-27, 2000.

Carolina Vittoria Beccari, Daniel E. Gonsor, and Marian Neamtu. RAGS: Rational geometric splines for surfaces of arbitrary topology. Computer Aided Geometric Design, 31(2):97-110, 2014.

Georges-Pierre Bonneau and Stefanie Hahmann. Flexible $G^{1}$ interpolation of quad meshes. Graphical Models, 76(6):669-681, 2014 .

Michel Bercovier and Tanya Matskewich. Smooth Bézier Surfaces over Unstructured Quadrilateral Meshes. Lecture Notes of the Unione Matematica Italiana. Springer International Publishing, 2017.

Ahmed Blidia, Bernard Mourrain, and Nelly Villamizar. $\mathrm{G}^{1}$-smooth splines on quad meshes with 4-split macro-patch elements. Computer Aided Geometric Design, 52:106-125, 2017.

C. L. Chan, C. Anitescu, and T. Rabczuk. Isogeometric analysis with strong multipatch $C^{1}$-coupling. Computer Aided Geometric Design, 62:294-310, 2018.

E. Catmull and J. Clark. Recursively generated B-spline surfaces on arbitrary topological meshes. Computer-Aided Design, 10(6):350-355, 1978.

Doo-Yeoun Cho, Kyu-Yeul Lee, and Tae-Wan Kim. Interpolating $G^{1}$ Bézier surfaces over irregular curve networks for ship hull design. Computer-Aided Design, 38(6):641-660, 2006.

Annabelle Collin, Giancarlo Sangalli, and Thomas Takacs. Analysis-suitable $G^{1}$ multi-patch parametrizations for $C^{1}$ isogeometric spaces. Computer Aided Geometric Design, 47:93-113, 2016.

Jianhua Fan and Jörg Peters. On smooth bicubic surfaces from quad meshes. In International Symposium on Visual Computing, pages 87-96. Springer, 2008.

Xianfeng Gu, Ying He, and Hong Qin. Manifold splines. Graphical Models, 68(3):237-254, 2006.

David Groisser and Jörg Peters. Matched Gk-constructions always yield Ck-continuous isogeometric elements. Computer Aided Geometric Design, 34:67-72, 2015.

Günther Greiner. Variational design and fairing of spline surfaces. Comput. Graph. Forum, 13:143-154, 08 1994.

Stefanie Hahmann, Georges-Pierre Bonneau, and Baptiste Caramiaux. Bicubic $G^{1}$ interpolation of irregular quad meshes using a 4-split. In International Conference on Geometric Modeling and Processing, pages 17-32. Springer, 2008.

Ying He, Kexiang Wang, Hongyu Wang, Xianfeng Gu, and Hong Qin. Manifold T-spline. In International Conference on Geometric Modeling and Processing, pages 409-422. Springer, 2006.

Mario Kapl, Florian Buchegger, Michel Bercovier, and Bert Jüttler. Isogeometric analysis with geometrically continuous functions on planar multi-patch geometries. Computer Methods in Applied Mechanics and Engineering, 316:209-234, 2017.

Mario Kapl, Giancarlo Sangalli, and Thomas Takacs. Dimension and basis construction for analysis-suitable $G^{1}$ two-patch parameterizations. Computer Aided Geometric Design, 52-53:75-89, 2017.

Mario Kapl, Giancarlo Sangalli, and Thomas Takacs. An isogeometric $C^{1}$ subspace on unstructured multi-patch planar domains. Computer Aided Geometric Design, 69:55-75, 2019.

Mario Kapl, Vito Vitrih, Bert Jüttler, and Katharina Birner. Isogeometric analysis with geometrically continuous functions on two-patch geometries. Computers \& Mathematics with Applications, 70(7):1518-1538, 2015.

Kestutis Karčiauskas, Thien Nguyen, and Jörg Peters. Generalizing bicubic splines for modeling and IGA with irregular layout. Computer-Aided Design, 70:23-35, 2016.

Kestutis Karčiauskas and Jörg Peters. Improved shape for refinable surfaces with singularly parameterized irregularities. Computer-Aided Design, 90:191-198, 2017. 
Kestutis Karčiauskas and Jörg Peters. Refinable $G^{1}$ functions on $G^{1}$ free-form surfaces. Computer Aided Geometric Design, 54:61-73, 2017.

Kestutis Karčiauskas and Jörg Peters. Refinable bi-quartics for design and analysis. Computer-Aided Design, 102:204-214, 2018.

Charles Loop. A $G^{1}$ triangular spline surface of arbitrary topological type. Computer Aided Geometric Design, 11(3):303-330, 1994.

Charles Loop. Smooth spline surfaces over irregular meshes. In Proceedings of the 21st Annual Conference on Computer Graphics and Interactive Techniques, pages 303-310. ACM, 1994.

Ming-Jun Lai and Larry L. Schumaker. Spline Functions on Triangulations. Cambridge University Press, April 2007.

M. Majeed and F. Cirak. Isogeometric analysis using manifold-based smooth basis functions. Computer Methods in Applied Mechanics and Engineering, 316:547-567, April 2017.

Bernard Mourrain, Raimundas Vidunas, and Nelly Villamizar. Dimension and bases for geometrically continuous splines on surfaces of arbitrary topology. Computer Aided Geometric Design, 45:108-133, 2016.

Jörg Peters. Smooth interpolation of a mesh of curves. Constructive Approximation, 7(1):221-246, 1991.

Jörg Peters. Biquartic $C^{1}$-surface splines over irregular meshes. Computer-Aided Design, 27(12):895-903, 1995.

Jörg Peters. Geometric continuity. Handbook of computer aided geometric design, pages 193-229, 2002.

Jörg Peters and Jianhua Fan. On the complexity of smooth spline surfaces from quad meshes. Computer Aided Geometric Design, 27(1):96-105, 2010.

Hartmut Prautzsch. Freeform splines. Computer Aided Geometric Design, 14(3):201-206, 1997.

Ulrich Reif. Biquadratic G-spline surfaces. Computer Aided Geometric Design, 12(2):193-205, 1995.

Ramon F. Sarraga. $\mathrm{G}^{1}$ interpolation of generally unrestricted cubic Bézier curves. Computer Aided Geometric Design, 4(12):23-39, 1987.

Ramon F. Sarraga. Errata: $G^{1}$ interpolation of generally unrestricted cubic Bézier curves. Computer Aided Geometric Design, $6(2): 167-171,1989$.

Alvaro J. Fuentes Suárez and Evelyne Hubert. Scaffolding skeletons using spherical Voronoï diagrams. Electronic Notes in Discrete Mathematics, 62:45 - 50, 2017. LAGOS'17 - IX Latin and American Algorithms, Graphs and Optimization.

Wei-hua Tong and Tae-wan Kim. High-order approximation of implicit surfaces by triangular spline surfaces. Computer-Aided Design, 41(6):441-455, 2009.

Deepesh Toshniwal, Hendrik Speleers, and Thomas J. R. Hughes. Smooth cubic spline spaces on unstructured quadrilateral meshes with particular emphasis on extraordinary points: Geometric design and isogeometric analysis considerations. Computer Methods in Applied Mechanics and Engineering, 327:411-458, 2017.

Elif Tosun and Denis Zorin. Manifold-based surfaces with boundaries. Computer Aided Geometric Design, 28(1):1-22, 2011.

Ruimin Wang, Ligang Liu, Zhouwang Yang, Kang Wang, Wen Shan, Jiansong Deng, and Falai Chen. Construction of Manifolds via Compatible Sparse Representations. ACM Transactions on Graphics, 35(2):1-10, February 2016.

Meng Wu, Bernard Mourrain, André Galligo, and Boniface Nkonga. $\mathrm{H}^{1}$-parametrizations of complex planar physical domains in isogeometric analysis. Computer Methods in Applied Mechanics and Engineering, 318:296-318, 2017.

Wenping Wang, Helmut Pottmann, and Yang Liu. Fitting B-spline Curves to Point Clouds by Curvature-based Squared Distance Minimization. ACM Trans. Graph., 25(2):214-238, April 2006.

Lexing Ying and Denis Zorin. A simple manifold-based construction of surfaces of arbitrary smoothness. In SIGGRAPH 'O4, pages 271-275. ACM Press, 2004.

Andre Weil. Foundations of Algebraic Geometry. American Mathematical Society. 1946. Colloquium Publications. 